\title{
An evolutionary trade-off between parasite virulence and dispersal at experimental invasion fronts
}

\author{
Louise Nørgaard ${ }^{1}$, Giacomo Zilio² ${ }^{2}$ Camille Saade ${ }^{2}$, Claiire Gougat-Barbera ${ }^{2}$, Matthew \\ Hall $^{1,1}$, Emanuel Fronhofer ${ }^{2}$, and Oliver Kaltz ${ }^{2}$ \\ ${ }^{1}$ Monash University \\ ${ }^{2}$ University of Montpellier
}

August 24, 2020

\begin{abstract}
Changing environments and habitat structure likely affect eco-evolutionary processes involved in the spatial spread of disease. Exploitative parasites are predicted to evolve in highly connected populations or in expanding epidemics. However, many parasites rely on host dispersal to reach new populations, potentially causing conflict between local transmission and global spread. We performed experimental range expansions in interconnected microcosms of the protozoan Paramecium caudatum, allowing natural dispersal of hosts infected with the bacterial parasite Holospora undulata. Parasites from range front treatments were less virulent and interfered less with host dispersal, but also invested less in horizontal transmission than parasites from range cores. An epidemiological model fitted on experimental time-series data confirmed this trade-off between dispersal adaptation and transmission, so far rarely considered in theoretical models. Our study illustrates the importance of the ecology and evolution of dispersal-related traits in spatial non-equilibrium scenarios, including emerging diseases, metapopulations or biological invasions.
\end{abstract}

\section{Hosted file}

Noergaard_et_al_main_text-2.pdf available at https://authorea.com/users/353397/articles/ 477311-an-evolutionary-trade-off-between-parasite-virulence-and-dispersal-atexperimental-invasion-fronts 